\title{
Refinement of the crystal structure of Sb-I, at 2.22 GPa
}

\author{
L. G. Akselrud ${ }^{\mathrm{I}, \mathrm{II}}$, M. Hanfland ${ }^{\mathrm{III}}$ and U. Schwarz ${ }^{*, \mathrm{II}}$ \\ I Lviv State University, Kyryla and Mefodija St. 6, 79005 Lviv, Ukraine \\ II Max-Planck-Institut für Chemische Physik fester Stoffe, Nöthnitzer Str. 40, D-01187 Dresden, Germany \\ ${ }^{\text {III }}$ ESRF, BP 220, F-38043 Grenoble, France
}

Received November 25, accepted and available on-line November 26, 2003, CSD-No. 409754

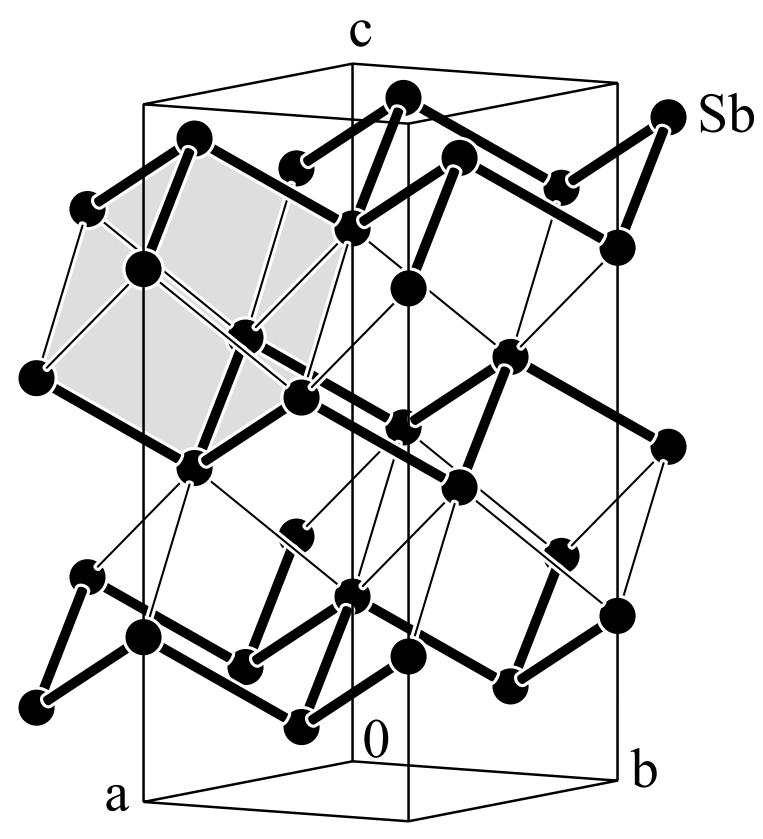

\section{Abstract}

Sb, trigonal, $R \overline{3} m$ (No. 166), $a=4.2687(2) \AA, c=10.9244(6) \AA$, $V=172.4 \AA^{3}, Z=6, R(P)=0.066, R(I)=0.059, T=295 \mathrm{~K}$, $P=2.22 \mathrm{GPa}$.

\section{Source of material}

Antimony of $99.999 \%$ purity (ABCR GmbH, Germany) was used for the experiments. Polycrystalline samples were obtained by grinding of antimony shots at ambient conditions.

\section{Experimental details}

Angle-dispersive X-ray powder diffraction experiments were performed on ID-9 at ESRF using an image plate detector. The powdered particles were placed in a gasketed diamond anvil high-pressure cell using a small sphere of ruby for pressure calibration and a 4:1 mixture of ethanol and methanol as a pressure transmitting medium.

\section{Discussion}

Structural parameters of antimony at elevated pressures up to $8.5 \mathrm{GPa}$ were determined earlier by means of single crystal X-ray diffraction in diamond anvil cells [1]. We re-refined the parameter values in order to gain a quantitative measure for the reliability of full profile refinements using powder patterns measured with synchrotron radiation. The result of the least squares procedure is within three standard deviations identical to the values obtained by single crystal diffraction methods $(z=0.2358(6)$ at $P=1.5$ GPa and $z=0.2379(6)$ at $P=2.66 \mathrm{GPa}$ [1]) . The resulting interatomic distances of $3 \times 2.895(1) \AA$ and $3 \times 3.253(1) \AA$ support the finding that the rhombohedral low-pressure phase approaches a simple cubic arrangement with increasing pressures [1,2].

Table 1. Data collection and handling.

$\begin{array}{ll}\text { Powder: } & \text { black, size } 5-10 \mu \mathrm{m} \\ \text { Wavelength: } & \text { synchrotron radiation }(0.41844 \AA) \\ \mu: & 75.0 \mathrm{~cm}^{-1} \\ \text { Diffractometer: } & \text { MAR3450 } \\ 2 \theta_{\text {ma }}, \text { stepwidth } & 24.9^{\circ}, 0.01 \\ N(\text { points })_{\text {measured: }} & 2304 \\ N(h k l)_{\text {measured }} & 35 \\ N(\text { param })_{\text {refined }}: & 4 \\ \text { Programs: } & \text { WinCSD [3], ImageIntegrator [4] }\end{array}$

\section{References}

1. Schiferl, D.; Cromer, D. T.; Jamieson, J. C.: Structure determinations on Sb up to $85 \times 10^{2}$ MPa. Acta Crstallogr. B37 (1981) 807-810.

2. Schwarz, U.; Akselrud, L. G.; Rosner, H.; Ormeci, A.; Grin, Yu.: Structure and stability of the modulated phase Sb-II. Phys. Rev. B67 (2003) 214101

3. Akselrud, L. G.; Grin, Yu. N.; Zavalii, P. Yu.; Pecharskii, V. K.: WinCSD - Universal program package for single crystal and/or powder structure data treatment. Windows version 10-2003.

4. Akselrud, L. G.; Schwarz, U.: ImageIntegrator: A program for treatment of image-plate powder-diffraction data. Version 10-2003. MPI CPfS, Dresden, Germany 2003.

Table 2. Atomic coordinates and displacement parameters (in $\AA^{2}$ ).

\begin{tabular}{lllllllllll}
\hline Atom & Site & $x$ & $y$ & $z$ & $U_{11}$ & $U_{22}$ & $U_{33}$ & $U_{12}$ & $U_{13}$ & $U_{23}$ \\
\hline $\mathrm{Sb}$ & $6 c$ & 0 & 0 & $0.2362(1)$ & $0.038(5)$ & $U_{11}$ & $0.046(9)$ & $U_{11} / 2$ & 0 & 0 \\
\hline
\end{tabular}

\footnotetext{
* Correspondence author (e-mail: schwarz@ cpfs.mpg.de)
} 